\title{
Some reverse Hölder inequalities with Specht's ratio on time scales
}

\author{
A. A. El-Deeb ${ }^{a}$, H. A. Elsennary ${ }^{a, b}$, Wing-Sum Cheung ${ }^{c, *}$ \\ ${ }^{a}$ Department of Mathematics, Faculty of Science, Al-Azhar University, Nasr City (11884), Cairo, Egypt. \\ ${ }^{b}$ Department of Mathematics, Faculty of Engineering, Sinai University, El Arish (45615), North Sinai, Egypt. \\ ${ }^{c}$ Department of Mathematics, The University of Hong Kong, Pokfulam Road, Hong Kong.
}

Communicated by M. Bohner

\begin{abstract}
In this article, we investigate some new reverse Hölder-type inequalities on an arbitrary time scale via the diamond- $\alpha$ dynamic integral, which is defined as a linear combination of the delta and nabla integrals. These inequalities extend some known dynamic inequalities on time scales, unify and extend some continuous inequalities and their corresponding discrete analogues.
\end{abstract}

Keywords: Dynamic inequalities of Hölder type, analysis techniques, time scales, Specht's ratio.

2010 MSC: 26D10, 26D15, 26D20, 34A12, 34A40.

(C)2018 All rights reserved.

\section{Introduction}

We start from the famous Young inequality $[5,10,15,16]$ :

$$
(1-v) a+v b \geqslant a^{1-v} b^{v}
$$

for positive numbers $\mathrm{a}, \mathrm{b}$ and $v \in[0,1]$. The inequality (1.1) is also called $v$-weighted arithmetic-geometric mean inequality and its reverse inequality with Specht's ratio was given in [1] by

$$
S\left(\frac{a}{b}\right) a^{1-v} b^{v} \geqslant(1-v) a+v b
$$

for positive numbers $\mathrm{a}, \mathrm{b}$ and $v \in[0,1]$, where the Specht's ratio $[9,20]$ is defined by

$$
S(h)=\frac{h^{\frac{1}{h-1}}}{e \log h^{\frac{1}{h-1}}} \quad(h>0, h \neq 1) .
$$

\footnotetext{
*Corresponding author

Email addresses: ahmedeldeeb@azhar.edu.eg (A. A. El-Deeb), hamza.abderabou@su.edu.eg (H. A. Elsennary), wscheung@hku.hk (Wing-Sum Cheung)
}

doi: $10.22436 /$ jnsa.011.04.01

Received: 2017-08-18 Revised: 2017-11-24 Accepted: 2018-01-18 
Inequality (1.2) can be written as:

$$
S\left(\frac{a}{b}\right) a^{\frac{1}{p}} b^{\frac{1}{q}} \geqslant \frac{a}{p}+\frac{b}{q}
$$

for positive numbers $a, b$ and $\frac{1}{p}+\frac{1}{q}=1$ with $p>1$.

We review some properties of the Specht's ratio. See $[9,20,22]$ for the proof and details:

- $S(1)=1$ and $S(h)=S(1 / h)>1$ for $h>0$.

- $S(h)$ is a monotone increasing function on $(1, \infty)$.

- $S(h)$ is a monotone decreasing function on $(0,1)$.

The well-known classical Hölder's inequality $[5,10,15,16]$ states that

$$
\sum_{i=1}^{n} a_{i} b_{i} \leqslant\left(\sum_{i=1}^{n} a_{i}^{p}\right)^{\frac{1}{p}}\left(\sum_{i=1}^{n} b_{i}^{q}\right)^{\frac{1}{q}}
$$

where $\left\{a_{i}\right\}_{i=1}^{n}$ and $\left\{b_{i}\right\}_{i=1}^{n}$ are nonnegative real sequences and $p, q \in \mathbb{R}$ with $p>1$ and $\frac{1}{p}+\frac{1}{q}=1$.

The integral version of Hölder's inequality is

$$
\int_{a}^{b}|f(x) g(x)| d x \leqslant\left(\int_{a}^{b}|f(x)|^{p} d x\right)^{\frac{1}{p}}\left(\int_{a}^{b}|g(x)|^{q} d x\right)^{\frac{1}{q}},
$$

where $f$ and $g$ are continuous functions on $[a, b]$ and $p, q \in \mathbb{R}$ with $p>1$ and $\frac{1}{p}+\frac{1}{q}=1$.

Using delta integral, Agarwal et al. [2] have given the time scale version of Hölder's inequality, namely,

$$
\int_{a}^{b}|f(x) g(x)| \Delta x \leqslant\left(\int_{a}^{b}|f(x)|^{p} \Delta x\right)^{\frac{1}{p}}\left(\int_{a}^{b}|g(x)|^{q} \Delta x\right)^{\frac{1}{q}},
$$

where $a, b \in \mathbb{T}^{\kappa}$ and $f, g \in C_{r d}\left([a, b]_{\mathbb{T}}, \mathbb{R}\right)$ and $p, q \in \mathbb{R}$ with $p>1$ and $\frac{1}{p}+\frac{1}{q}=1$.

Recently Zhao and Cheung [27] proved a reverse Hölder's inequality with Specht's ratio. They showed that, for any positive continuous functions $f$ and $g$ on $[a, b]$, if $\frac{1}{p}+\frac{1}{q}=1$ with $p>1$,

$$
\int_{a}^{b} S\left(\frac{Y f^{p}(x)}{X g^{q}(x)}\right) f(x) g(x) d x \geqslant\left(\int_{a}^{b} f^{p}(x) d x\right)^{\frac{1}{p}}\left(\int_{a}^{b} g^{q}(x) d x\right)^{\frac{1}{q}},
$$

where

$$
X=\int_{a}^{b} f^{p}(x) d x \quad \text { and } \quad Y=\int_{a}^{b} g^{q}(x) d x .
$$

By replacing $f^{p}$ and $g^{q}$ by $f$ and $g$, respectively, inequality (1.4) can be rewritten as:

$$
\int_{a}^{b} S\left(\frac{Y f(x)}{X g(x)}\right) f^{\frac{1}{p}}(x) g^{\frac{1}{q}}(x) d x \geqslant\left(\int_{a}^{b} f(x) d x\right)^{\frac{1}{p}}\left(\int_{a}^{b} g(x) d x\right)^{\frac{1}{q}},
$$

where

$$
X=\int_{a}^{b} f(x) d x \quad \text { and } \quad Y=\int_{a}^{b} g(x) d x .
$$

Hölder's inequality is an important tool in different branches of modern mathematics such as classical real and complex analysis, numerical analysis, probability and differential equations. Since its discovery, it has been studied widely and has been generalized on many ways, see [1, 4, 13, 14, 17, 18, 24, 25]. Some reverse versions of Hölder's inequality were established in [8, 21, 23, 26]. 
The theory of time scales, which has recently received a lot of attention, was initiated by Hilger in his $\mathrm{PhD}$ thesis in order to unify discrete and continuous analysis [11]. The general idea is to prove a result for a dynamic equation or a dynamic inequality where the domain of the unknown function is a so called time scale $\mathbb{T}$, which may be an arbitrary closed subset of the real numbers $\mathbb{R}$ see $[6,7]$. The three most popular examples of calculus on time scales are differential calculus, difference calculus, and quantum calculus (see [12]), i.e., when $\mathbb{T}=\mathbb{R}, \mathbb{T}=\mathbb{N}$ and $\mathbb{T}=q^{N_{0}}=\left\{q^{t}: t \in N_{0}\right\}$ where $q>1$. The book on the subject of time scales by Bohner and Peterson [6] summarizes and organizes much of time scale calculus. During the past decade a number of dynamic inequalities has been established by some authors which are motivated by practical problems, for example, when studying the behavior of solutions of certain class of dynamic equations on a time scale $\mathbb{T}$, see $[2,3,6]$, and the references cited therein.

In this paper, we prove some reverse Hölder inequalities with Specht's ratio using the diamond- $\alpha$ derivative on time scales and give their continuous and discrete analogues. The diamond- $\alpha$ derivative reduces to the standard $\Delta$ derivative for $\alpha=1$, or the standard $\nabla$ derivative for $\alpha=0$. We refer the reader to [19] for an account of the calculus corresponding to the diamond- $\alpha$ dynamic derivative. The paper is organized as follows. In Section 2, some basic concepts of the calculus on time scales and useful lemmas are introduced. In Section 3, we state and prove the main results.

\section{Preliminaries and lemmas on time scales}

First, we will present some preliminaries on calculus of time scales and some universal symbols used in this article. Throughout the paper $\mathbb{R}$ denotes the set of real numbers, $\mathbb{R}_{0}^{+}=[0, \infty)$, and $\mathbb{Z}$ denotes the set of integers. A time scale $\mathbb{T}$ is an arbitrary nonempty closed subset of the real numbers. We assume throughout that $\mathbb{T}$ has the topology that it inherits from the standard topology on the real numbers $\mathbb{R}$. For $t \in \mathbb{T}$, first we define the forward jump operator $\sigma: \mathbb{T} \rightarrow \mathbb{T}$ by:

$$
\sigma(t):=\inf \{s \in \mathbb{T}: s>t\},
$$

and second, the backward jump operator $\rho: \mathbb{T}: \rightarrow \mathbb{T}$ by:

$$
\rho(t):=\sup \{s \in \mathbb{T}: s<t\},
$$

where $\sup \emptyset=\inf \mathbb{T}$. A point $t \in \mathbb{T}$ with $\inf \mathbb{T}<t<\sup \mathbb{T}$ is said to be left-dense if $\rho(t)=t$ and is right-dense if $\sigma(t)=t$, points that are simultaneously right-dense and left-dense are said to be dense, $t$ is left-scattered if $\rho(t)<t$ and right-scattered if $\sigma(t)>t$, points that are simultaneously right-scattered and left-scattered are said to be isolated. A function $g: \mathbb{T} \rightarrow \mathbb{R}$ is said to be right-dense continuous (rd-continuous) provided $g$ is continuous at right-dense points and at left-dense points in $\mathbb{T}$, left hand limits exist and are finite. The set of all such rd-continuous functions is denoted by $C_{r d}(\mathbb{T})$. A function $\mathrm{f}: \mathbb{T} \rightarrow \mathbb{R}$ is said to be left-dense continuous (ld-continuous) provided $\mathrm{f}$ is continuous at left-dense points and at right-dense points in $\mathbb{T}$, right-hand limits exist and are finite. The set of all such ld-continuous functions is denoted by $C_{l d}(\mathbb{T})$.

The forward and backward graininess functions $\mu$ and $\nu$ for a time scale $\mathbb{T}$ are defined by $\mu(t):=$ $\sigma(t)-t$ and $\nu(t):=t-\rho(t)$, respectively.

Given a time scale $\mathbb{T}$, we introduce the sets $\mathbb{T}^{k}, \mathbb{T}_{K}$, and $\mathbb{T}_{k}^{k}$ as follows. If $\mathbb{T}$ has a left-scattered maximum $t_{1}$, then $\mathbb{T}^{k}=\mathbb{T}-\left\{t_{1}\right\}$, otherwise $\mathbb{T}^{k}=\mathbb{T}$. If $\mathbb{T}$ has a right-scattered minimum $t_{2}$, then $\mathbb{T}_{\kappa}=\mathbb{T}-\left\{t_{2}\right\}$, otherwise $\mathbb{T}_{\kappa}=\mathbb{T}$. Finally $\mathbb{T}_{\kappa}^{k}=\mathbb{T}^{\kappa} \cap \mathbb{T}_{\kappa}$.

Let $f: \mathbb{T} \rightarrow \mathbb{R}$ be a real valued function on a time scale $\mathbb{T}$. Then $\forall t \in \mathbb{T}^{k}$, we define $f^{\Delta}(t)$ to be the number (if it exists) with the property that for any given $\varepsilon>0$ there is a neighborhood $U$ of $t$ such that $\forall$ $\mathrm{s} \in \mathrm{U}$,

$$
\left|[f(\sigma(t))-f(s)]-f^{\Delta}(t)[\sigma(t)-s]\right| \leqslant \varepsilon|\sigma(t)-s|, \quad \forall s \in U
$$

In this case we say that $f$ is delta differentiable on $\mathbb{T}^{k}$ provided $f^{\Delta}(t)$ exists for all $t \in \mathbb{T}_{k}$. Similarly, $\forall$ $t \in \mathbb{T}_{k}$, we define $f^{\nabla}(t)$ to be the number (if it exists) with the property that for any given $\varepsilon>0$ there is a 
neighborhood $\mathrm{U}$ of $\mathrm{t}$ such that $\forall s \in \mathrm{U}$,

$$
\left|[f(\rho(t))-f(s)]-f^{\nabla}(t)[\rho(t)-s]\right| \leqslant \varepsilon|\rho(t)-s|, \quad \forall s \in U .
$$

In this case we say that $f$ is nabla differentiable on $\mathbb{T}_{K}$ provided $f^{\nabla}(t)$ exists for all $t \in \mathbb{T}_{k}$. For $f: \mathbb{T} \rightarrow \mathbb{R}$, we define the function $f^{\sigma}: \mathbb{T} \rightarrow \mathbb{R}$ by $f^{\sigma}=f(\sigma), \forall t \in \mathbb{T}$, that is $f^{\sigma}(t)=(f \circ \sigma)(t)$. Similarly, we define the function $f^{\rho}: \mathbb{T} \rightarrow \mathbb{R}$ by $f^{\rho}=f(\rho), \forall t \in \mathbb{T}$, that is $f^{\rho}(t)=(f \circ \rho)(t)$. A time scale $\mathbb{T}$ is said to be regular if the following two conditions are satisfied simultaneously: (1) $\sigma(\rho(t))=t$, and (2) $\rho(\sigma(t))=t, \forall$ $t \in \mathbb{T}$. The product and the quotient rules for the derivative of the product $f g$ and the quotient $f / g$ (where $g(t) g^{\sigma}(t) \neq 0$, here $\left.g^{\sigma}=g \circ \sigma\right)$ of two differentiable functions $f$ and $g$, are given as the following:

$$
(f g)^{\Delta}(t)=f^{\Delta}(t) g(t)+f(\sigma(t)) g^{\Delta}(t)=f(t) g^{\Delta}(t)+f^{\Delta}(t) g(\sigma(t)),
$$

and

$$
\left(\frac{f}{g}\right)^{\Delta}(t)=\frac{f^{\Delta}(t) g(t)-f(t) g^{\Delta}(t)}{g(t) g(\sigma(t))}
$$

A function $F: \mathbb{T} \rightarrow \mathbb{R}$, is called a delta antiderivative of $f: \mathbb{T} \rightarrow \mathbb{R}$ provided that $F^{\Delta}(t)=f(t)$ holds $\forall$ $t \in \mathbb{T}^{k}$, then the delta integral of $f$ is defined by

$$
\int_{a}^{b} f(t) \Delta t=F(b)-F(a) .
$$

A function $G: \mathbb{T} \rightarrow \mathbb{R}$ is called a nabla antiderivative of $g: \mathbb{T} \rightarrow \mathbb{R}$ provided that $G^{\nabla}(t)=g(t)$ holds $\forall \mathrm{t} \in \mathbb{T}_{\kappa}$, then the nabla integral of $\mathrm{g}$ is defined by

$$
\int_{a}^{b} g(t) \nabla t=G(b)-G(a) .
$$

We will frequently use the following useful relations between calculus on time scales $\mathbb{T}$ and differential calculus on $\mathbb{R}$, difference calculus on $\mathbb{Z}$, and quantum calculus on $q_{0}^{\mathbb{N}}$. Note that if

(i) $\mathbb{T}=\mathbb{R}$, then

$$
\sigma(t)=t, \mu(t)=0, f^{\Delta}(t)=f^{\prime}(t), \int_{a}^{b} f(t) \Delta t=\int_{a}^{b} f(t) d t
$$

(ii) if $\mathbb{T}=\mathbb{Z}$, then

$$
\sigma(t)=t+1, \mu(t)=1, f^{\Delta}(t)=\Delta f(t), \int_{a}^{b} f(t) \Delta t=\sum_{t=a}^{b-1} f(t)
$$

(iii) if $\mathbb{T}=h \mathbb{Z}, h>0$, then

$$
\sigma(t)=t+h, \mu(t)=h, \text { and } \int_{a}^{b} f(t) \Delta t=\sum_{k=0}^{\frac{b-a-h}{h}} f(a+k h) h ;
$$

(v) if $\mathbb{T}=q^{\mathbb{N}_{0}}=\left\{t: t=q^{k}, k \in \mathbb{N}_{0}, q>1\right\}$, then

$$
\sigma(t)=q t, \mu(t)=(q-1) t, \int_{a}^{b} f(t) \Delta t=(q-1) \sum_{k=\log _{q}(a)}^{\log _{q}(b)-1} q^{k} f\left(q^{k}\right), \forall a, b \in q^{\mathbb{N}_{0}}
$$

It can be shown (see [6]) that if $g \in C_{r d}(\mathbb{T})$, then the Cauchy integral $G(t):=\int_{t_{0}}^{t} g(s) \Delta s$ exists, $t_{0} \in \mathbb{T}$, and satisfies $G^{\Delta}(t)=g(t), t \in \mathbb{T}$. An infinite integral is defined as

$$
\int_{a}^{\infty} f(t) \Delta t=\lim _{b \rightarrow \infty} \int_{a}^{b} f(t) \Delta t
$$


Lemma 2.1 ([6, Theorem 1.76 on page 28]). If $\mathrm{f}^{\Delta}(\mathrm{t}) \geqslant 0$, then $\mathrm{f}(\mathrm{t})$ is nondecreasing.

Lemma 2.2 ([6, Theorem 1.90. Chain Rule on page 32]). Let $f: \mathbb{R} \rightarrow \mathbb{R}$ be continuously differentiable and suppose $\mathrm{g}: \mathbb{T} \rightarrow \mathbb{R}$ is delta differentiable. Then $\mathrm{f} \circ \mathrm{g}: \mathbb{T} \rightarrow \mathbb{R}$ is delta differentiable and the formula

$$
(\mathrm{f} \circ \mathrm{g})^{\Delta}(\mathrm{t})=\left\{\int_{0}^{1}\left[\mathrm{f}^{\prime}\left(\mathrm{g}(\mathrm{t})+\mathrm{h} \mu(\mathrm{t}) \mathrm{g}^{\Delta}(\mathrm{t})\right)\right] \mathrm{dh}\right\} \mathrm{g}^{\Delta}(\mathrm{t}),
$$

holds.

Lemma 2.3 ([6, Exercise 1.23 on page 9]). Assume that $\mathrm{f}: \mathbb{T} \rightarrow \mathbb{R}$ is delta differentiable at $\mathrm{t} \in \mathbb{T}^{\mathrm{k}}$. Then

$$
\left(f^{n}\right)^{\Delta}(x)=\left\{\sum_{k=0}^{n-1} f^{k}(x)\left[f^{\sigma}(x)\right]^{n-1-k}\right\} f^{\Delta}(x) .
$$

Lemma 2.4 ([6, Theorem 1.16 on page 5]). Assume that $\mathrm{f}: \mathbb{T} \rightarrow \mathbb{R}$ is delta differentiable at $\mathrm{t} \in \mathbb{T}^{\mathrm{k}}$. Then

$$
f^{\sigma}(x)=f(x)+\mu(x) f^{\Delta}(x) .
$$

Now, we briefly introduce the diamond- $\alpha$ dynamic derivative and the diamond- $\alpha$ dynamic integral, and we refer the reader to [19] for a comprehensive development of the calculus of the diamond- $\alpha \mathrm{dy}-$ namic derivative and the diamond- $\alpha$ dynamic integration.

Let $\mathbb{T}$ be a time scale and $f(t)$ be differentiable on $\mathbb{T}$ in the $\Delta$ and $\nabla$ senses. For $t \in T$, we define the diamond- $\alpha$ dynamic derivative $f^{\diamond \alpha}(t)$ by

$$
f^{\diamond \alpha}(t)=\alpha f^{\Delta}(t)+(1-\alpha) f^{\nabla}(t), \quad 0 \leqslant \alpha \leqslant 1 .
$$

Thus $\mathrm{f}$ is diamond- $\alpha$ differentiable if and only if $\mathrm{f}$ is $\Delta$ and $\nabla$ differentiable. The diamond- $\alpha$ derivative reduces to the standard $\Delta$ derivative for $\alpha=1$, or the standard $\nabla$ derivative for $\alpha=0$. On the other hand, it represents a weighted dynamic derivative for $\alpha \in(0,1)$. Furthermore, the combined dynamic derivative offers a centralized derivative formula on any uniformly discrete time scale $\mathbb{T}$ when $\alpha=1 / 2$.

Let $f, g: \mathbb{T} \rightarrow \mathbb{R}$ be diamond- $\alpha$ differentiable at $t \in \mathbb{T}$. Then

(i) $f+g: \mathbb{T} \rightarrow \mathbb{R}$ is diamond- $\alpha$ differentiable at $t \in T$ with

$$
(f+g)^{\diamond \alpha}(t)=f^{\diamond \alpha}(t)+g{ }^{\diamond}(t) ;
$$

(ii) for any constant $c$, $c f: \mathbb{T} \rightarrow \mathbb{R}$ is diamond- $\alpha$ differentiable at $t \in T$ with

$$
(c f)^{\diamond \alpha}(t)=c f^{\diamond \alpha}(t)
$$

(ii) $\mathrm{fg}: \mathbb{T} \rightarrow \mathbb{R}$ is diamond- $\alpha$ differentiable at $t \in T$ with

$$
(f g)^{\nabla_{\alpha}}(t)=f^{\diamond \alpha}(t) g(t)+\alpha f^{\sigma}(t) g^{\Delta}(t)+(1-\alpha) f^{\rho}(t) g^{\nabla}(t) .
$$

Let $a, t \in \mathbb{T}$, and $h: \mathbb{T} \rightarrow \mathbb{R}$. Then the diamond- $\alpha$ integral from $a$ to $t$ of $h$ is defined by

$$
\int_{a}^{t} h(\tau) \nabla_{\alpha} \tau=\alpha \int_{a}^{t} h(\tau) \Delta \tau+(1-\alpha) \int_{a}^{t} h(\tau) \nabla \tau, \quad 0 \leqslant \alpha \leqslant 1 .
$$

We may notice that the $\nabla_{\alpha}$ integral is a combined $\Delta$ and $\nabla$ integral. In general, we do not have

$$
\left(\int_{a}^{t} h(\tau) \nabla_{\alpha} \tau\right)^{\nabla_{\alpha}}=h(t), \quad t \in \mathbb{T}
$$

Let $a, b, t \in \mathbb{T}, c \in \mathbb{R}$. Then

(i) $\int_{a}^{t}[f(\tau)+g(\tau)] \diamond_{\alpha} \tau=\int_{a}^{t} f(\tau) \diamond_{\alpha} \tau+\int_{a}^{t} g(\tau) \diamond_{\alpha} \tau$;

(ii) $\int_{a}^{t} c f(\tau) \diamond_{\alpha} \tau=c \int_{a}^{t} f(\tau) \diamond_{\alpha} \tau$;

(iii) $\int_{a}^{t} f(\tau) \diamond_{\alpha} \tau=\int_{a}^{b} f(\tau) \diamond_{\alpha} \tau+\int_{b}^{t} f(\tau) \diamond_{\alpha} \tau$;

(iv) $\int_{a}^{t} f(\tau) \diamond_{\alpha} \tau=-\int_{t}^{a} f(\tau) \diamond_{\alpha} \tau$;

(v) if $f(t) \leqslant g(t)$ for all $\tau \in[a, b]_{\mathbb{T}}$, then $\int_{a}^{b} f(\tau) \diamond_{\alpha} \tau \leqslant \int_{a}^{b} g(\tau) \diamond_{\alpha} \tau$.

Now we are ready to state and prove our main results. 


\section{Main results}

Throughout this section, $\mathbb{T}$ is an arbitrary time scale, neither $f \equiv 0, g \equiv 0$ nor $h \equiv 0$ and we assume that all integrals converge.

Theorem 3.1. Let $\mathrm{a}, \mathrm{b} \in \mathbb{T}_{\mathrm{K}}^{\mathrm{k}}$ and $\mathrm{f}, \mathrm{g}, \mathrm{h} \in \mathrm{C}\left([\mathrm{a}, \mathrm{b}]_{\mathbb{T}}, \mathbb{R}_{0}^{+}\right)$such that $\mathrm{f}^{\mathrm{p}}$ and $\mathrm{g}^{\mathrm{q}}$ are $\diamond_{\alpha}$-integrable on $[\mathrm{a}, \mathrm{b}]_{\mathbb{T}}$. If $\frac{1}{\mathrm{p}}+\frac{1}{\mathrm{q}}=1$ with $\mathrm{p}>1$, then

$$
\int_{a}^{b} S\left(\frac{Y f^{p}(t)}{X g^{q}(t)}\right) h(t) f(t) g(t) \diamond_{\alpha} t \geqslant\left(\int_{a}^{b} h(t) f^{p}(t) \diamond_{\alpha} t\right)^{\frac{1}{p}}\left(\int_{a}^{b} h(t) g^{q}(t) \diamond_{\alpha} t\right)^{\frac{1}{q}}
$$

where

$$
X=\int_{a}^{b} h(t) f^{p}(t) \diamond_{\alpha} t \quad \text { and } \quad Y=\int_{a}^{b} h(t) g^{q}(t) \diamond_{\alpha} t
$$

and $\mathrm{S}($.$) is the Specht's ratio.$

Proof. Letting

$$
a=\frac{h(t) f^{p}(t)}{X} \quad \text { and } \quad b=\frac{h(t) g^{q}(t)}{Y}
$$

in (1.3), we get

$$
S\left(\frac{Y f^{p}(t)}{X g^{q}(t)}\right) \frac{h(t) f(t) g(t)}{X^{\frac{1}{p}} Y^{\frac{1}{q}}} \geqslant \frac{1}{p} \frac{h(t) f^{p}(t)}{X}+\frac{1}{q} \frac{h(t) g^{q}(t)}{Y} .
$$

By integrating both sides over $t$ from $a$ to $b$, we obtain

$$
\frac{\int_{a}^{b} S\left(\frac{Y f^{p}(t)}{X g^{q}(t)}\right) h(t) f(t) g(t) \nabla_{\alpha} t}{X^{\frac{1}{p}} Y^{\frac{1}{q}}} \geqslant \frac{1}{p} \frac{\int_{a}^{b} h(t) f^{p}(t) \diamond_{\alpha} t}{X}+\frac{1}{q} \frac{\int_{a}^{b} h(t) g^{q}(t) \diamond_{\alpha} t}{Y}=\frac{1}{p}+\frac{1}{q}=1,
$$

which is the desired inequality. This completes the proof.

Corollary 3.2. When $\mathrm{h}(\mathrm{t})=1$ in Theorem 3.1, we get

$$
\int_{a}^{b} S\left(\frac{Y f^{p}(t)}{X g^{q}(t)}\right) f(t) g(t) \nabla_{\alpha} t \geqslant\left(\int_{a}^{b} f^{p}(t) \diamond_{\alpha} t\right)^{\frac{1}{p}}\left(\int_{a}^{b} g^{q}(t) \nabla_{\alpha} t\right)^{\frac{1}{q}} .
$$

As a special case of Theorem 3.1, if $\mathbb{T}=\mathbb{R}$ and using the relations (2.1), we get the following continuous result.

Corollary 3.3. Let $\mathrm{f}, \mathrm{g}, \mathrm{h} \in \mathrm{C}\left([\mathrm{a}, \mathrm{b}], \mathbb{R}_{0}^{+}\right)$. If $\frac{1}{\mathrm{p}}+\frac{1}{\mathrm{q}}=1$ with $\mathrm{p}>1$, then

$$
\int_{a}^{b} S\left(\frac{Y f^{p}(t)}{X g^{q}(t)}\right) h(t) f(t) g(t) d t \geqslant\left(\int_{a}^{b} h(t) f^{p}(t) d t\right)^{\frac{1}{p}}\left(\int_{a}^{b} h(t) g^{q}(t) d t\right)^{\frac{1}{q}}
$$

where

$$
X=\int_{a}^{b} h(t) f^{p}(t) d t \quad \text { and } \quad Y=\int_{a}^{b} h(t) g^{q}(t) d t .
$$

Remark 3.4. If we take $h(t)=1$ in Corollary 3.3 we obtain Theorem 1.4 due to Zhao and Cheung [27].

As a special case of Theorem 3.1, if $\mathbb{T}=\mathbb{Z}$ and using the relations (2.2), we get the following discrete result. 
Corollary 3.5. Let $\mathrm{f}(\mathrm{n}), \mathrm{g}(\mathrm{n})$, and $\mathrm{h}(\mathrm{n})$ be nonnegative real sequences. If $\frac{1}{\mathrm{p}}+\frac{1}{\mathrm{q}}=1$ with $\mathrm{p}>1$, then

$$
\sum_{n=a}^{b-1} S\left(\frac{Y f^{p}(n)}{X g^{q}(n)}\right) h(n) f(n) g(n) \geqslant\left(\sum_{n=a}^{b-1} h(n) f^{p}(n)\right)^{\frac{1}{p}}\left(\sum_{n=a}^{b-1} h(n) g^{q}(n)\right)^{\frac{1}{q}},
$$

where

$$
X=\sum_{n=a}^{b-1} h(n) f^{p}(n) \quad \text { and } \quad Y=\sum_{n=a}^{b-1} h(n) g^{q}(n) .
$$

Theorem 3.6. Let $\mathrm{a}, \mathrm{b} \in \mathbb{T}_{\mathrm{K}}^{\mathrm{K}}$ and $\mathrm{f}, \mathrm{g}, \mathrm{h} \in \mathrm{C}\left([\mathrm{a}, \mathrm{b}]_{\mathbb{T}}, \mathbb{R}_{0}^{+}\right)$. If $\frac{1}{\mathrm{p}}+\frac{1}{\mathrm{q}}=1$ with $\mathrm{p}<0$ or $\mathrm{q}<0$, then

$$
\left(\int_{a}^{b} S\left(\frac{Y f^{p-1}(t)}{X g(t)}\right) g^{q}(t) \diamond \alpha t\right)^{\frac{1}{q}}\left(\int_{a}^{b} f^{p}(t) \nabla_{\alpha} t\right)^{\frac{1}{p}} \geqslant\left(\int_{a}^{b} f(t) g(t) \nabla_{\alpha} t\right),
$$

where

$$
X=\int_{a}^{b} f^{p}(t) \diamond_{\alpha} t \quad \text { and } \quad Y=\int_{a}^{b} f(t) g(t) \diamond_{\alpha} t .
$$

Proof. Assume that $\mathrm{p}<0$ (the proof when $\mathrm{q}<0$ is similar). Set $\mathrm{P}=\frac{-\mathrm{p}}{\mathrm{q}}$ and $\mathrm{Q}=\frac{1}{\mathrm{q}}$. Then $\frac{1}{\mathrm{P}}+\frac{1}{\mathrm{Q}}=1$ with $\mathrm{P}>1$ and $\mathrm{Q}>1$. Let $\mathrm{F}, \mathrm{G} \in \mathrm{C}\left([\mathrm{a}, \mathrm{b}], \mathbb{R}^{+}\right)$such that $\mathrm{F}^{\mathrm{P}}$ and $\mathrm{G}^{\mathrm{Q}}$ are $\diamond_{\alpha}$-integrable on $[a, b]_{\mathbb{T}}$. Then from (3.1) we have

$$
\int_{a}^{b} S\left(\frac{F^{P} \int_{a}^{b} G^{Q}(t) \diamond_{\alpha} t}{G Q \int_{a}^{b} F^{P}(t) \diamond_{\alpha} t}\right) F(t) G(t) \diamond_{\alpha} t \geqslant\left(\int_{a}^{b} F^{P}(t) \diamond_{\alpha} t\right)^{\frac{1}{P}}\left(\int_{a}^{b} G^{Q}(t) \diamond_{\alpha} t\right)^{\frac{1}{Q}} .
$$

Setting $F(t)=f^{-q}$ and $G(t)=f^{q} g^{q}$ in the last inequality, we get

$$
\int_{a}^{b} S\left(\frac{f^{p}(t) \int_{a}^{b} f(t) g(t) \diamond_{\alpha} t}{f(t) g(t) \int_{a}^{b} f^{p}(t) \diamond_{\alpha} t}\right) g^{q}(t) \diamond_{\alpha} t \geqslant\left(\int_{a}^{b} f^{p}(t) \diamond_{\alpha} t\right)^{\frac{-q}{p}}\left(\int_{a}^{b} f(t) g(t) \diamond_{\alpha} t\right)^{q},
$$

or

$$
\int_{a}^{b} S\left(\frac{Y f^{p-1}(t)}{X g(t)}\right) g^{q}(t) \diamond_{\alpha} t\left(\int_{a}^{b} f^{p}(t) \nabla_{\alpha} t\right)^{\frac{q}{p}} \geqslant\left(\int_{a}^{b} f(x) g(t) \nabla_{\alpha} t\right)^{q}
$$

Therefore

$$
\left(\int_{a}^{b} S\left(\frac{Y f^{p-1}(t)}{X g(t)}\right) g^{q}(t) \nabla_{\alpha} t\right)^{\frac{1}{q}}\left(\int_{a}^{b} f^{p}(t) \nabla_{\alpha} t\right)^{\frac{1}{p}} \geqslant\left(\int_{a}^{b} f(t) g(t) \nabla_{\alpha} t\right) .
$$

This completes the proof.

As a special case of Theorem 3.6, if $\mathbb{T}=\mathbb{R}$ and using the relations (2.1), we get the following continuous result.

Corollary 3.7. Let $\mathrm{f}, \mathrm{g}, \mathrm{h} \in \mathrm{C}\left([\mathrm{a}, \mathrm{b}], \mathbb{R}_{0}^{+}\right)$. If $\frac{1}{\mathrm{p}}+\frac{1}{\mathrm{q}}=1$ with $\mathrm{p}<0$ or $\mathrm{q}<0$, then

$$
\left(\int_{a}^{b} S\left(\frac{Y f^{p-1}(t)}{X g(t)}\right) g^{q}(t) d t\right)^{\frac{1}{q}}\left(\int_{a}^{b} f^{p}(t) d t\right)^{\frac{1}{p}} \geqslant\left(\int_{a}^{b} f(t) g(t) d t\right),
$$

where

$$
X=\int_{a}^{b} f^{p}(t) d t \quad \text { and } \quad Y=\int_{a}^{b} f(t) g(t) d t .
$$

As a special case of Theorem 3.6, if $\mathbb{T}=\mathbb{Z}$ and using the relations (2.2), we get the following discrete result. 
Corollary 3.8. Let $\mathrm{f}(\mathrm{n})$ and $\mathrm{g}(\mathrm{n})$ be nonnegative real sequences. If $\frac{1}{\mathrm{p}}+\frac{1}{\mathrm{q}}=1$ with $\mathrm{p}>1$, then

$$
\left(\sum_{n=a}^{b-1} S\left(\frac{Y f^{p-1}(n)}{X g(n)}\right) g^{q}(n)\right)^{\frac{1}{q}}\left(\sum_{n=a}^{b-1} f^{p}(n)\right)^{\frac{1}{p}} \geqslant\left(\sum_{n=a}^{b-1} f(n) g(n)\right),
$$

where

$$
X=\sum_{n=a}^{b-1} f^{p}(n) \quad \text { and } \quad Y=\sum_{n=a}^{b-1} f(n) g(n) .
$$

Theorem 3.9. Let $\mathrm{a}, \mathrm{b} \in \mathbb{T}_{\mathrm{k}}^{\mathrm{k}}$ and $\mathrm{f}, \mathrm{g} \in \mathrm{C}\left([\mathrm{a}, \mathrm{b}]_{\mathbb{T}}, \mathbb{R}_{0}^{+}\right)$such that $0<\mathrm{m} \leqslant \frac{\mathrm{f}(\mathrm{t})}{\mathrm{g}(\mathrm{t})} \leqslant \mathrm{M}<\infty$ for all $\mathrm{t} \in[\mathrm{a}, \mathrm{b}]_{\mathbb{T}}$. If $\frac{1}{p}+\frac{1}{q}=1$ with $p>1$, then

$$
\int_{a}^{b} S\left(\frac{Y f(t)}{X g(t)}\right) f^{\frac{1}{p}}(t) g^{\frac{1}{q}}(t) \diamond_{\alpha} t \geqslant \frac{m^{\frac{1}{p^{2}}}}{M^{\frac{1}{q^{2}}}}\left(\int_{a}^{b} f^{\frac{1}{q}}(t) g^{\frac{1}{p}}(t) \diamond_{\alpha} t\right),
$$

where

$$
X=\int_{a}^{b} f(t) \diamond_{\alpha} t \quad \text { and } \quad Y=\int_{a}^{b} g(t) \diamond_{\alpha} t
$$

Proof. From (1.5), we have

$$
\int_{a}^{b} S\left(\frac{Y f(t)}{X g(t)}\right) f^{\frac{1}{p}}(t) g^{\frac{1}{q}}(t) \diamond_{\alpha} t \geqslant\left(\int_{a}^{b} f(t) \diamond_{\alpha} t\right)^{\frac{1}{p}}\left(\int_{a}^{b} g(t) \diamond_{\alpha} t\right)^{\frac{1}{q}},
$$

that is,

$$
\int_{a}^{b} S\left(\frac{Y f(t)}{X g(t)}\right) f^{\frac{1}{p}}(t) g^{\frac{1}{q}}(t) \diamond_{\alpha} t \geqslant\left(\int_{a}^{b} f^{\frac{1}{p}}(t) f^{\frac{1}{q}}(t) \diamond_{\alpha} t\right)^{\frac{1}{p}}\left(\int_{a}^{b} g^{\frac{1}{p}}(t) g^{\frac{1}{q}}(t) \nabla_{\alpha} t\right)^{\frac{1}{q}} .
$$

Since $f^{\frac{1}{p}}(t) \geqslant m^{\frac{1}{p}} g^{\frac{1}{p}}(t)$ and $g^{\frac{1}{q}}(t) \geqslant M^{\frac{-1}{q}} f^{\frac{1}{q}}(t)$, then

$$
\int_{a}^{b} S\left(\frac{Y f(t)}{X g(t)}\right) f^{\frac{1}{p}} g^{\frac{1}{q}} \nabla_{\alpha} t \geqslant \frac{m^{\frac{1}{p^{2}}}}{M^{\frac{1}{q^{2}}}}\left(\int_{a}^{b} f^{\frac{1}{q}}(t) g^{\frac{1}{p}}(t) \diamond_{\alpha} t\right)^{\frac{1}{p}}\left(\int_{a}^{b} f^{\frac{1}{q}}(t) g^{\frac{1}{p}}(t) \nabla_{\alpha} t\right)^{\frac{1}{q}},
$$

and so

$$
\int_{a}^{b} S\left(\frac{Y f(t)}{X g(t)}\right) f^{\frac{1}{p}}(t) g^{\frac{1}{q}}(t) \diamond_{\alpha} t \geqslant \frac{m^{\frac{1}{p^{2}}}}{M^{\frac{1}{q^{2}}}}\left(\int_{a}^{b} f^{\frac{1}{q}}(t) g^{\frac{1}{p}}(t) \diamond_{\alpha} t\right) .
$$

Hence, the inequality (3.2) is proved.

As a special case of Theorem 3.9, if $\mathbb{T}=\mathbb{R}$ and using the relations (2.1), we get the following continuous result.

Corollary 3.10. Let $\mathrm{f}, \mathrm{g} \in \mathrm{C}\left([\mathrm{a}, \mathrm{b}], \mathbb{R}_{0}^{+}\right)$such that $0<\mathrm{m} \leqslant \frac{\mathrm{f}(\mathrm{t})}{\mathrm{g}(\mathrm{t})} \leqslant \mathrm{M}<\infty$ for all $\mathrm{t} \in[\mathrm{a}, \mathrm{b}]$. If $\frac{1}{\mathrm{p}}+\frac{1}{\mathrm{q}}=1$ with $p>1$, then

$$
\int_{a}^{b} S\left(\frac{Y f(t)}{X g(t)}\right) f^{\frac{1}{p}}(t) g^{\frac{1}{q}}(t) d t \geqslant \frac{m^{\frac{1}{p^{2}}}}{M^{\frac{1}{q^{2}}}}\left(\int_{a}^{b} f^{\frac{1}{q}}(t) g^{\frac{1}{p}}(t) d t\right),
$$

where

$$
X=\int_{a}^{b} f(t) d t \quad \text { and } \quad Y=\int_{a}^{b} g(t) d t .
$$

As a special case of Theorem 3.9, if $\mathbb{T}=\mathbb{Z}$ and using the relations (2.2), we get the following discrete result. 
Corollary 3.11. Let $\mathrm{f}(\mathrm{n})$ and $\mathrm{g}(\mathrm{n})$ be nonnegative real sequences such that $0<\mathrm{m} \leqslant \frac{\mathrm{f}(\mathrm{n})}{\mathrm{g}(\mathrm{n})} \leqslant M<\infty$. If $\frac{1}{\mathrm{p}}+\frac{1}{\mathrm{q}}=1$ with $\mathrm{p}>1$, then

$$
\sum_{n=a}^{b-1} S\left(\frac{Y f(n)}{X g(n)}\right) f^{\frac{1}{p}}(n) g^{\frac{1}{q}}(n) \geqslant \frac{m^{\frac{1}{p^{2}}}}{M^{\frac{1}{q^{2}}}}\left(\sum_{n=a}^{b-1} f^{\frac{1}{q}}(n) g^{\frac{1}{p}}(n)\right),
$$

where

$$
X=\sum_{n=a}^{b-1} f(n) \quad \text { and } \quad Y=\sum_{n=a}^{b-1} g(n) .
$$

Theorem 3.12. Let $\mathrm{a}, \mathrm{b} \in \mathbb{T}_{\mathrm{K}}^{\mathrm{K}}$ and $\mathrm{f}, \mathrm{g} \in \mathrm{C}\left([\mathrm{a}, \mathrm{b}]_{\mathbb{T}}, \mathbb{R}_{0}^{+}\right)$such that $\mathrm{f}^{\mathrm{p}}$ and $\mathrm{g}^{\mathrm{q}}$ are integrable on $[\mathrm{a}, \mathrm{b}]$. If $\frac{1}{\mathrm{p}}+\frac{1}{\mathrm{q}}=1$ with $\mathrm{p}>1$ and $0<\mathrm{m} \leqslant \frac{\mathrm{f}^{\mathrm{p}}(\mathrm{t})}{\mathrm{g}^{\mathrm{q}}(\mathrm{t})} \leqslant \mathrm{M}<\infty, \forall \mathrm{t} \in[\mathrm{a}, \mathrm{b}]_{\mathbb{T}}$, then

$$
\int_{a}^{b} S\left(\frac{Y f^{p}(t)}{X g^{q}(t)}\right) f(t) g(t) \diamond_{\alpha} t \geqslant\left(\frac{m}{M}\right)^{\frac{1}{p q}}\left(\int_{a}^{b} f(t) g(t) \nabla_{\alpha} t\right),
$$

where

$$
X=\int_{a}^{b} f^{p}(t) \diamond_{\alpha} t \quad \text { and } \quad Y=\int_{a}^{b} g^{q}(t) \diamond_{\alpha} t
$$

Proof. From (1.4), we have

$$
\int_{a}^{b} S\left(\frac{Y f^{p}(t)}{X g^{q}(t)}\right) f(t) g(t) \diamond \alpha t \geqslant\left(\int_{a}^{b} f^{p}(t) \diamond \alpha t\right)^{\frac{1}{p}}\left(\int_{a}^{b} g^{q}(t) \diamond \alpha t\right)^{\frac{1}{q}} .
$$

Since $m \leqslant \frac{f^{p}(t)}{g^{q}(t)}$, then we have $g(t) \leqslant m^{\frac{-1}{q}} f^{\frac{p}{q}}(t)$, and so

$$
f(t) g(t) \leqslant m^{\frac{-1}{q}} f^{\frac{p}{q}+1}(t)=m^{\frac{-1}{q}} f^{\frac{p+q}{q}}(t)=m^{\frac{-1}{q}} f^{p}(t) .
$$

Therefore

$$
\left(\int_{a}^{b} f^{p}(t) \diamond_{\alpha} t\right)^{\frac{1}{p}} \geqslant m^{\frac{1}{p q}}\left(\int_{a}^{b} f(t) g(t) \diamond_{\alpha} t\right)^{\frac{1}{p}} .
$$

Also since $\frac{f^{p}(t)}{g^{q}(t)} \leqslant M$, then we have $f(t) \leqslant M^{\frac{1}{p}} g^{\frac{q}{p}}(t)$, and so

$$
f(t) g(t) \leqslant M^{\frac{1}{p}} g^{\frac{q}{p}+1}(t)=M^{\frac{1}{p}} g^{\frac{q+p}{p}}(t)=M^{\frac{1}{p}} g^{q}(t) .
$$

Therefore

$$
\left(\int_{a}^{b} g^{p}(t) \diamond_{\alpha} t\right)^{\frac{1}{q}} \geqslant M^{\frac{-1}{p q}}\left(\int_{a}^{b} f(t) g(t) \diamond_{\alpha} t\right)^{\frac{1}{q}} .
$$

Combining (3.3), (3.4), and (3.5), we get

$$
\int_{a}^{b} S\left(\frac{Y f^{p}(t)}{X g^{q}(t)}\right) f(t) g(t) \diamond_{\alpha} t \geqslant\left(\frac{m}{M}\right)^{\frac{1}{p q}}\left(\int_{a}^{b} f(t) g(t) \diamond_{\alpha} t\right)^{\frac{1}{p}}\left(\int_{a}^{b} f(t) g(t) v\right)^{\frac{1}{q}},
$$

that is,

$$
\int_{a}^{b} S\left(\frac{Y f^{p}(t)}{X g^{q}(t)}\right) f(t) g(t) \diamond_{\alpha} t \geqslant\left(\frac{m}{M}\right)^{\frac{1}{p q}}\left(\int_{a}^{b} f(t) g(t) \diamond_{\alpha} t\right) .
$$

This completes the proof.

As a special case of Theorem 3.12, if $\mathbb{T}=\mathbb{R}$ and using the relations (2.1), we get the following continuous result. 
Corollary 3.13. Let $\mathrm{f}, \mathrm{g} \in \mathrm{C}\left([\mathrm{a}, \mathrm{b}], \mathbb{R}_{0}^{+}\right)$. If $\frac{1}{\mathrm{p}}+\frac{1}{\mathrm{q}}=1$ with $\mathrm{p}>1$ and $0<\mathrm{m} \leqslant \frac{\mathrm{f}^{\mathrm{p}}(\mathrm{t})}{\mathrm{g}^{\mathrm{q}}(\mathrm{t})} \leqslant \mathrm{M}<\infty$ for all $\mathrm{t} \in[\mathrm{a}, \mathrm{b}]$, then

$$
\int_{a}^{b} S\left(\frac{Y f^{p}(t)}{X g^{q}(t)}\right) f(t) g(t) d t \geqslant\left(\frac{m}{M}\right)^{\frac{1}{p q}}\left(\int_{a}^{b} f(t) g(t) d t\right),
$$

where

$$
X=\int_{a}^{b} f^{p}(t) d t \quad \text { and } \quad Y=\int_{a}^{b} g^{q}(t) d t .
$$

As a special case of Theorem 3.12, if $\mathbb{T}=\mathbb{Z}$ and using the relations (2.2), we get the following discrete result.

Corollary 3.14. Let $\mathrm{f}(\mathrm{n})$ and $\mathrm{g}(\mathrm{n})$ be nonnegative real sequences such that $0<\mathrm{m} \leqslant \frac{\mathrm{f}^{\mathrm{p}}(\mathrm{n})}{\mathrm{g}^{\mathrm{q}}(\mathrm{n})} \leqslant M<\infty$. If $\frac{1}{p}+\frac{1}{q}=1$ with $p>1$, then

$$
\sum_{n=a}^{b-1} S\left(\frac{Y f^{p}(n)}{X g^{q}(n)}\right) f(n) g(n) \geqslant\left(\frac{m}{M}\right)^{\frac{1}{p q}}\left(\sum_{n=a}^{b-1} f(n) g(n)\right)
$$

where

$$
X=\sum_{n=a}^{b-1} f^{p}(n) \quad \text { and } \quad Y=\sum_{n=a}^{b-1} g^{q}(n) .
$$

Now we give a two-dimensional reverse Hölder's inequality with Specht's ratio.

Theorem 3.15. Let $\mathrm{a}, \mathrm{b} \in \mathbb{T}_{\mathrm{K}}^{\mathrm{K}}$ and $\mathrm{f}, \mathrm{g}, \mathrm{h} \in \mathrm{C}\left([\mathrm{a}, \mathrm{b}]_{\mathbb{T}} \times[\mathrm{a}, \mathrm{b}]_{\mathbb{T}}, \mathbb{R}_{0}^{+}\right)$. If $\frac{1}{\mathrm{p}}+\frac{1}{\mathrm{q}}=1$ with $\mathrm{p}>1$, then

$$
\begin{aligned}
\int_{a}^{b} \int_{a}^{b} S\left(\frac{Y f^{p}(t, s)}{X g^{q}(t, s)}\right) h(t, s) f(t, s) g(t, s) \nabla_{\alpha} s \diamond_{\alpha} t & \geqslant\left(\int_{a}^{b} \int_{a}^{b} h(t, s) f^{p}(t, s) \nabla_{\alpha} s \nabla_{\alpha} t\right)^{\frac{1}{p}} \\
& \times\left(\int_{a}^{b} \int_{a}^{b} h(t, s) g^{q}(t, s) \nabla_{\alpha} s \nabla_{\alpha} t\right)^{\frac{1}{q}},
\end{aligned}
$$

where

$$
X=\int_{a}^{b} \int_{a}^{b} h(t, s) f^{p}(t, s) \diamond_{\alpha} s \diamond_{\alpha} t \quad \text { and } \quad Y=\int_{a}^{b} \int_{a}^{b} h(t, s) g^{q}(t, s) \diamond_{\alpha} s \diamond_{\alpha} t .
$$

Proof. Letting

$$
a=\frac{h(t, s) f^{p}(t, s)}{\int_{a}^{b} \int_{a}^{b} h(t, s) f^{p}(t, s) \diamond_{\alpha} s \diamond_{\alpha} t} \quad \text { and } \quad b=\frac{h(t, s) g^{q}(t, s)}{\int_{a}^{b} \int_{a}^{b} h(t, s) g^{q}(t, s) \nabla_{\alpha} s \triangleright_{\alpha} t}
$$

in (1.3) we get

$$
\frac{S\left(\frac{Y f^{p}(t, s)}{X g^{q}(t, s)}\right) h(t, s) f(t, s) g(t, s)}{X^{\frac{1}{p}} Y^{\frac{1}{q}}} \geqslant \frac{1}{p} \frac{h(t, s) f^{p}(t, s)}{\left.p \int_{a}^{b} \int_{a}^{b} h(t, s) f^{p}(t, s)\right\rangle_{\alpha} s \nabla_{\alpha} t}+\frac{1}{q} \frac{h(t, s) g^{q}(t, s)}{\left.\int_{a}^{b} \int_{a}^{b} h(t, s) g^{q}(t, s)\right\rangle_{\alpha} s \diamond_{\alpha} t} .
$$

By integrating over $s$ from $a$ to $b$ first and then integrating the resulting inequality over $t$ from $a$ to $b$, we obtain

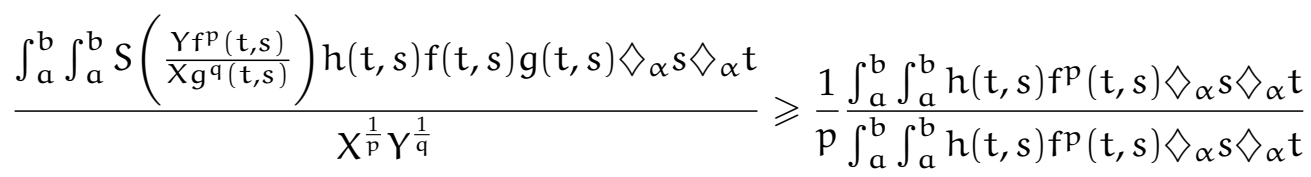

$$
\begin{aligned}
& +\frac{1}{q} \frac{\int_{a}^{b} \int_{a}^{b} h(t, s) g^{q}(t, s) \diamond_{\alpha} s \diamond_{\alpha} t}{\int_{a}^{b} \int_{a}^{b} h(t, s) g^{q}(t, s) \diamond_{\alpha} s \diamond_{\alpha} t}=\frac{1}{p}+\frac{1}{q}=1
\end{aligned}
$$

and the desired inequality follows. This completes the proof. 
As a special case of Theorem 3.15, if $\mathbb{T}=\mathbb{R}$ and using the relations (2.1), we get the following continuous result.

Corollary 3.16. Let $\mathrm{f}, \mathrm{g}, \mathrm{h} \in \mathrm{C}\left([\mathrm{a}, \mathrm{b}] \times[\mathrm{a}, \mathrm{b}], \mathbb{R}_{0}^{+}\right)$. If $\frac{1}{\mathrm{p}}+\frac{1}{\mathrm{q}}=1$ with $\mathrm{p}>1$, then

$$
\int_{a}^{b} \int_{a}^{b} S\left(\frac{Y f^{p}(t, s)}{X g^{q}(t, s)}\right) h(t, s) f(t, s) g(t, s) d s d t \geqslant\left(\int_{a}^{b} \int_{a}^{b} h(t, s) f^{p}(t, s) d s d t\right)^{\frac{1}{p}}\left(\int_{a}^{b} \int_{a}^{b} h(t, s) g^{q}(t, s) d s d t\right)^{\frac{1}{q}},
$$

where

$$
X=\int_{a}^{b} \int_{a}^{b} h(t, s) f^{p}(t, s) d s d t \quad \text { and } \quad Y=\int_{a}^{b} \int_{a}^{b} h(t, s) g^{q}(t, s) d s d t
$$

As another special case of Theorem 3.15, if $\mathbb{T}=\mathbb{Z}$ and using the relations (2.2), we get the following discrete result.

Corollary 3.17. Let $f(n, m), g(n, m)$, and $h(n, m)$ be nonnegative real sequences. If $\frac{1}{p}+\frac{1}{q}=1$ with $p>1$, then

$$
\begin{aligned}
\sum_{n=a}^{b-1} \sum_{m=a}^{b-1} S\left(\frac{Y^{p}(n, m)}{X g^{q}(n, m)}\right) h(n, m) f(n, m) g(n, m) \geqslant & \left(\sum_{n=a}^{b-1} \sum_{m=a}^{b-1} h(n, m) f^{p}(n, m)\right)^{\frac{1}{p}} \\
& \times\left(\sum_{n=a}^{b-1} \sum_{m=a}^{b-1} h(n, m) g^{q}(n, m)\right)^{\frac{1}{q}}
\end{aligned}
$$

where

$$
X=\sum_{n=a}^{b-1} \sum_{m=a}^{b-1} h(n, m) f^{p}(n, m) \quad \text { and } \quad Y=\sum_{n=a}^{b-1} \sum_{m=a}^{b-1} h(n, m) g^{q}(n, m)
$$

\section{Acknowledgment}

The last author's research was supported by a HKU Outstanding Teaching Award. The authors would like to thank the referees for their extremely careful reading and thoughtful suggestions.

\section{References}

[1] S. Abramovich, B. Mond, J. E. Pečarić, Sharpening Hölder's Inequality, J. Math. Anal. Appl., 196 (1995), $1131-1134$. 1,1

[2] R. Agarwal, M. Bohner, A. Peterson, Inequalities on time scales: a survey, Math. Inequal. Appl., 4 (2001), 535-557. 1, 1

[3] R. P. Agarwal, D. O’Regan, S. Saker, Dynamic inequalities on time scales, Springer, Cham, (2014). 1

[4] J. M. Aldaz, A stability version of Hölder's inequality, J. Math. Anal. Appl., 343 (2008), 842-852. 1

[5] E. F. Beckenbach, R. Bellman, Inequalities, Springer-Verlag, Berlin, (1961). 1, 1

[6] M. Bohner, A. Peterson, Dynamic equations on time scales, Birkhäuser Boston, Inc., Boston, (2001). 1, 2, 2.1, 2.2, 2.3, 2.4

[7] M. Bohner, A. Peterson, Advances in dynamic equations on time scales, Birkhäuser Boston, Inc., Boston, (2003). 1

[8] C. Borell, Inverse Hölder inequalities in one and several dimensions, J. Math. Anal. Appl., 41 (1973), 300-312. 1

[9] J. I. Fujii, S. Izumino, Y. Seo, Determinant for positive operators and Spechts theorem, Sci. Math., 1 (1998), 307-310. 1, 1

[10] G. H. Hardy, J. E. Littlewood, G. Pólya, In-equalities, Cambridge university press, Cambridge, (1952). 1, 1

[11] S. Hilger, Analysis on measure chains-a unied approach to continuous and discrete calculus, Results Math., 18 (1990), 18-56. 1

[12] V. Kac, P. Cheung, Quantum calculus, Springer-Verlag, New York, (2002). 1

[13] Y.-I. Kim, X. Yang, Generalizations and refinements of Hölders inequality, Appl. Math. Lett., 25 (2012), 1094-1097. 1

[14] E. G. Kwon, E. K. Bae, On a continuous form of Hölder inequality, J. Math. Anal. Appl., 343 (2008), 585-592. 1

[15] D. S. Mitrinović, Analytic inequalities, In cooperation with P. M. Vasić, Springer-Verlag, New York-Berlin, (1970). 1, 1

[16] D. S. Mitrinović, J. Pečarić, A. M. Fink, Classical and new inequalities in analysis, Kluwer Academic Publishers Group, Dordrecht, (1993). 1, 1 
[17] G. S. Mudholkar, M. Freimer, P. Subbaiah, An extension of Hölder inequality, J. Math. Anal. Appl., 102 (1984), 435-441. 1

[18] H. Qiang, Z. Hu, Generalizations of Hölder's and some related inequalities, Comput. Math. Appl., 61 (2011), $392-396$. 1

[19] Q. Sheng, M. Fadag, J. Henderson, J. M. Davis, An exploration of combined dynamic derivatives on time scales and their applications, Nonlinear Anal. Real World Appl., 7 (2006), 395-413. 1, 2

[20] W. Specht, Zur theorie der elementaren mittel, Math. Z, 74 (1960), 91-98. 1, 1

[21] J. Tian, Reversed version of a generalized sharp Hölder's inequality and its applications, Inform. Sci., 201 (2012), 61-69. 1

[22] M. Tominaga, Specht's ratio in the Young inequality, Sci. Math. Jpn., 55 (2002), 583-588. 1

[23] Y. V. Venkatesh, Converse Hölder inequality and the Lp-instability of non-linear time-varying feedback systems, Nonlinear Anal. Theor. Methods Appl., 12 (1988), 247-258. 1

[24] L. Wu, J. Sun, X. Ye, L. Zhu, Hölder type inequality for Sugeno integral, Fuzzy Sets and Systems, 161 (2010), 23372347. 1

[25] X. Yang, Hölder's inequality, Appl. Math. Lett., 16 (2003), 897-903. 1

[26] W. Yang, A functional generalization of diamond- $\alpha$ integral Hölder's inequality on time scales, Appl. Math. Lett., 23 (2010), 1208-1212. 1

[27] C.-J. Zhao, W. S. Cheung, Hölder's reverse inequality and its applications, Publ. Inst. Math., 99 (2016), 211-216. 1, 3.4 